\title{
A Multi-Factor Scheduling Algorithm for WIMAX Systems
}

\author{
Ahmed H. El-Shinnawy, Mohamed M. Elgazzar, and Amin M.Nassar
}

\begin{abstract}
This paper proposes a new scheduling algorithm for IEEE 802.16-2005 Broadband Wireless Metropolitan Area Networks in TDD mode. Unlike most scheduling algorithm which use one Qos parameter like Maximum Sustained Rate(MST), delay or priority to take the scheduling decision, the proposed algorithm uses all these parameters plus Jitter, Minimum rate, and class type. The algorithm put all these parameters in one polynomial, that gives priority to traffic session based on its class type, priority, and its lower QoS constrains. The priority of different sessions varies in time based on system dynamics, and the expected output of the system is a fair distribution for all session, with a maximization of throughput of priority traffic. In this work, a detailed simulation study is carried out for the proposed scheduling algorithm as well as comparing its performance with some known algorithms such as Proportional Fairness (PF)[12], Round Robin (RR), and Strict-Priority. Analyses and evaluation of the performance of the scheduler to support the different QoS classes is given as well. The simulation is carried out via the Opnet modeler simulator [13]. The results show the proposed algorithm is capable to handle different users' requirements under congestion conditions.
\end{abstract}

Index Terms-QoS, Opnet, OFDMA, scheduling algorithms, WIMAX

\section{INTRODUCTION}

Broadband wireless access (BWA) systems, [1], [2] are very flexible and easily deployable high-speed communication systems. BWA systems complement existing last mile wired networks such as cable modem and xDSL. IEEE 802.16 group aims to unify BWA solutions [1] A technical overview of IEEE 802.16 is provided in[1], [3]. The objective is to have an efficient use of radio resources while serving different types of data flows. These flows can have different constraints such as minimum traffic rate, maximum latency, and tolerated jitter.

The IEEE 802.16-2005 standard supports three different physical layers: 1) Single Carrier, 2) OFDM/TDMA and 3) OFDMA [1]. OFDMA physical layer is the most efficient and complex one[4]. In OFDMA each substation (SS) can receive some portions of the allocation for the combination of time and frequency so that the channel capacity is efficiently utilized. OFDMA outperforms the OFDM \& SC[4]. This research focuses only on OFDMA.

To support the different types of traffic with their various

Manuscript received February 23, 2012; revised April 28, 2012.

The authors are with the Faculty of Engineering Cairo University, Egypt requirements IEEE 802.16-2005 defines five QoS service classes: Unsolicited Grant Scheme (UGS), Extended Real Time Polling Service (ertPS), Real Time Polling Service (rtPS), Non Real Time Polling Service (nrtPS) and Best Effort Service (BE). Each of these class is identified with a specific parameters like maximum sustained rate (MST), maximum latency or tolerated jitter (the maximum delay variation) that suites the type of traffic that it serves. A details overview of these classes characteristics as well as types of traffic assigned to each class is illustrated in[1].

In IEEE 802.16-2005, the process in MAC Layer responsible for allocating resources for SSs (Subscriber Stations) and active flows is called the scheduling process. Unlike other parts of IEEE 802.16, scheduling was left for research to specify it. The optimal scheduling algorithm is still in open research area [6], [7], [8]. In this research a new scheduling algorithm is proposed to provide a better allocation of resources to different SSs in case of congestion, based on their QoS parameters and priority

The reminder of this paper is organized as follows. Section 2 provides a review for relevant work, simulated schedulers and problem formulation. In section 3 a detailed description of the proposed algorithm is given. Section 4 describes setup of the simulation environment Section 5 shows the results and output of simulation of the algorithm compared to other scheduling algorithms. Concluding remarks and directions for future work are given in section 6

\section{RELATED WORK AND PROBLEM FORMULATION}

\section{A. Related Work}

Scheduling techniques for WIMAX can be classified into two main categories: channel- unaware schedulers and channel-aware schedulers [4]. Channel-unaware schedulers use no information of the channel state condition in making the scheduling decision. The design of those schedulers varies based on the ultimate goal of the scheduler like, maximizing throughput or fair allocation of resources between different SSs. However, the main challenge facing researches is the distinctive characters of each of the QoS classes. No single queue algorithm can handle all QoS constrains simultaneously. For instance, no published researches show how to handle jitter over WiMAX, and most researches focuses on throughput rate or delay[4].

To overcome the obstacle above, many researches use Intra-class scheduling, where each class has a distinctive resource allocation mechanism that matches the requirements of the quality of service. Relation between inter classes is organized either on the bases of classpriority, where classes are served in the order UGS, ertPS, 
rtPS, nrtPS, BE, or on the bases of flow-priority, where highest priority flow is served first regardless of its class.

However, even within the same class, there are many constrains that can't be handled through one scheduler[4]. For instance, rtPS class is defined, by maximum sustained rate, minimum reserved rate, maximum latency and priority. Most existing scheduling algorithms give precedence to flows based on one or two of those parameters and neglect the remaining. For instance, RR, and all its variations WRR, WDRR[11], focuses only on the distribution of resource over all queues regardless of flow priority nor its minimum rate requirements. Other technique, like Max-Min Fair Allocation, focuses on giving priority to lowest data rate flow regardless of its priority or delay constrains. On the other hand, strict priority algorithms put more emphasis on high priority traffic, but cause other lower priority flows to starve.

To sum up, since the primary goal of a WIMAX scheduler is to assure the QoS requirements, the scheduler needs to support at least the five basic classes of services with QoS assurance.

\section{B. Problem Formulation,}

This research aims to design a scheduling algorithm a scheduling algorithm that is capable to consider all the Qos constrains of .all sessions. The algorithm should preserve the main characteristics of each class and be able discriminate throughput of each session based on its importance and priority as well.

The research focuses on handling rtPS \& nrtPS, Be classes, UGS and ertPS classes are very sensitive for delay/bandwidth variation and will be handled via strict priority, where their needs are served before any other class.

\section{PROPOSED ALGORITHM}

This proposed algorithm provides a mathematical model which aims to target the main parameters that govern the session behavior, which are iMaximum Sustained Rate(MST), Minimum reserved Rate(mRR), Maximum latency, Jitter, session priority and Class of service.

The first two parameter that controls class priority are the throughput rate compared to session MST and mRR.

Naturally throughput priority is reversal proportional to the rate

$$
\operatorname{Pr} i=1 / r
$$

where $r$ is the current throughput rate of the session

To normalize this priority value to be a function of throughput ratio from MST rather than actual throughput rate will convert priority ralation to be as follows:

$$
\operatorname{Pr} i=(R M-r) / R M
$$

Similarly, if the session mRR affects also the priority of the traffic as follow

$$
\operatorname{Pr} i=(R m-r) / R m
$$

In our design criteria for the algorithm, we adopt this two relations based on following factors:
1- The relation shouldn't be linear as shown eq. $2 \& 3$, it should be more none linear, where least throughput has much high priority than higher throughput.

2- We consider that achieving mRR has double the normal priority of achieving the MST.

3- The priority value should be normalized, between certain value, so when other parameters are considered they will not be overridden by these two parameters

Therefore, the suggested formula for throughput rate"r" and $\mathrm{mRR}$ and MST should be as follows:

$$
\mathrm{P}=\{a \times \exp ((R M-r) / R M)+2 \exp ((R m-r) / R m)-1\}
$$

where a in this equation is a parameter that represents the session priority

The expected priority function will have an output as shown in figure, where it represent the ration between the priority of the traffic, it actual throughput rate, in relevant to ration of $\mathrm{mRR}$ compared MST.

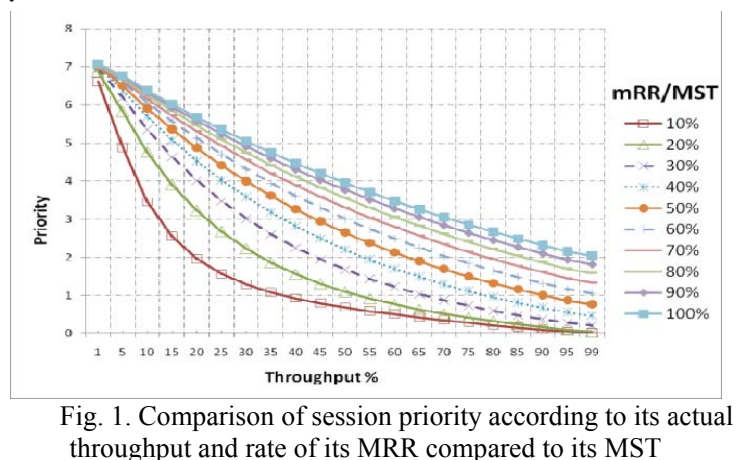

The next considered parameter is packet delay delay. Delay normally is related to two constrains, maximum latency and jitter. For maximum latency, the first constrain, it can be assumed that any delay value is suitable as long as it didn't preach the maximum latency. However the larger the delay value, the more probability it will exceed maximum latency. Therefore, priority of traffic relevant to delay should be exponential relation as follow:

$$
\operatorname{Pr} i=\exp (d / T) \wedge X
$$

where, $\mathrm{d}$ is the current delay, $\mathrm{T}$ is maximum latency, and $\mathrm{x}$ is a factor used to control the acceleration ratio of priority according to delay as in Fig. 2 in this research $x$ value is chosen to be "5" since it accelerate priority starting from $70 \%$ which gives the system a higher focus on the packet that are about to violate maximum latency and a suitable opportunity for the system to handle it before reaching maximum latency

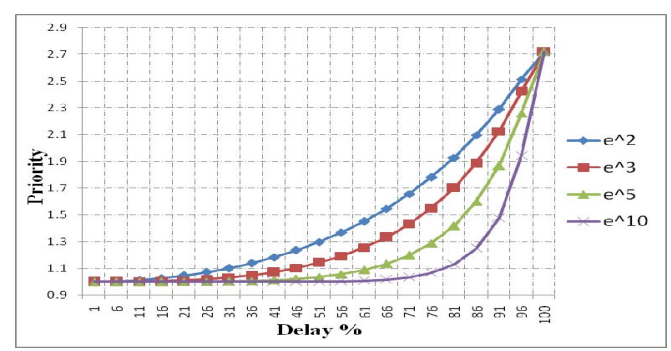

Fig. 2. Priority of traffic as a function of delay, and $x$ value. 
The second issue relevant to delay is the jitter, Formally, jitter is defined as a statistical variance of the RTP data packet inter-arrival time. Jitter acceptable value varies from protocol to protocol. So, unlike maximum latency jitter requires that data to be sent at a fixed intervals as much as possible. Or to be sent earlier or later that this fixed interval but within the acceptable jitter value. We think the Gaussian normal distribution function will be the best function to represent relation between priorities and jitter as follows:

$$
\operatorname{Pr} i=T / J * e((d-d 0) \wedge 2) /(2 J \wedge 2))
$$

where $\mathrm{J}$ is the acceptable jitter value, $\mathrm{d}_{0}$ is the expected constant interval delay (normally varies from $0.6 \sim 0.8$ of $\mathrm{T}$ the maximum latency. As it shown from the figure, the smaller the jitter value, the higher priority it has a $\mathrm{d}_{0}$ and the more variance in priority level around $\mathrm{d}_{0}$;

So base on the proposed relations provided in this section, the proposed priority relation will be sum of eqs 4,5,6. As indicated in equation 4 , priority of the session will only will be relevant to MST part of equation.

Finally class type can be consider as priority factor that affect the weight of each part of equation based on class type, but for the scope of this work all classes are assumed to have similar weight/effect of all parameters

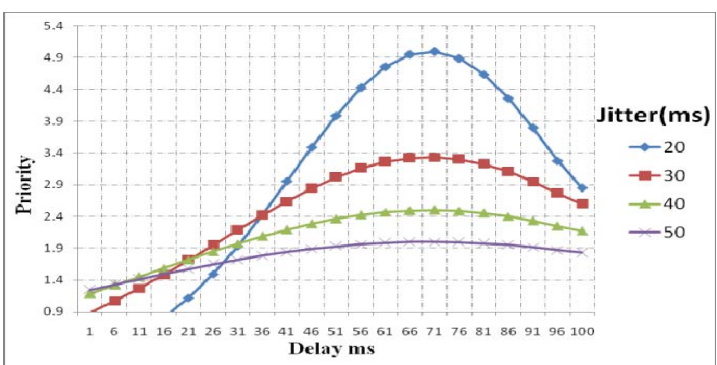

Fig. 3. Relation between jitter \& priority

\section{SiMUlation MODEL}

The overall goal of the simulation model is to analyze the behavior and performance of the proposed algorithm in a congested uplink domain The simulations have been performed using Opnet Modeler version 15.0 [14].The important parameters used to configure the PHY and MAC layers are summarized in Table I

TABLE I: Main PARAmeters of THE Simulation MOdel

\begin{tabular}{|l|l|}
\hline Model & Point to Multipoint \\
\hline WIMAX channel bandwidth & $=10 \mathrm{MHz}$ \\
\hline Frame duration & $5 \mathrm{~ms}$ \\
\hline Symbol Duration & $102.86 \mathrm{Micro}$ second \\
\hline $\mathrm{N}$ & $28 / 25$ \\
\hline Delta_f & $10.94 \mathrm{khz}$ \\
\hline Number of sub carriers & 1024 \\
\hline Frame structure & \\
\hline Preamble symbols & 1 symbol \\
\hline Dublexing technique & TDD \\
\hline Base Frequency & $2.5 \mathrm{GHZ}$ \\
\hline TTG & 106 micro second \\
\hline RTG & 60 micros second \\
\hline UL/DL Boundary & Fixed \\
\hline UL sub frame size & $=12$ slot \\
\hline DL sub frame size & $=32$ slot \\
\hline Initial ranging & $=2$ slot $* 6$ sub channel \\
\hline Contention slot & $=1$ slot 6 sub channel \\
\hline Initial coding rate & $3 / 4$ \\
\hline Initial modulation & $64 Q$ QM \\
\hline
\end{tabular}

The simulation assumes error-free channel since it makes it easier to prove assurance of QoS.

The simulation environment consists of one BS and (20 26) SSs operating in IEEE 802.16 PMP mode. There will be one service flow between each SS and the BS. Traffic flows classes and their configuration is indicated in Table II. The congestion condition in the system is conducted via increasing throughput load on the BS by increasing number of flows of a specific type (for example video priority traffic). In the simulation model divide the SSs in groups that have similar constrains, lime MST,mRR and to change only one parameter each time like maximum latency to test the system capabilities to responed to varies class constrains

TABLE II: SERVICE FLOWS

\begin{tabular}{|l|l|l|l|l|l|}
\hline Class & $\begin{array}{l}\text { Max. } \\
\text { rate } \\
\text { Kbps }\end{array}$ & $\begin{array}{l}\text { Min. } \\
\text { Rate } \\
\mathrm{kbps}\end{array}$ & Pri & $\begin{array}{l}\text { Traffic } \\
\text { Type }\end{array}$ & $\begin{array}{l}\text { No } \\
\text { of } \\
\text { SS }\end{array}$ \\
\hline UGS & 100 & 100 & N/A & Video & 2 \\
\hline RTP & $\begin{array}{l}384 \\
\sim 600\end{array}$ & 200 & 20 & Video & $2 \sim 8$ \\
\hline RTP & 384 & $200 \mathrm{~s}$ & 10 & Video & 2 \\
\hline nRTP & 384 & 200 & 20 & FTP & 1 \\
\hline nRTP & 384 & 200 & 10 & FTP & 2 \\
\hline nRTP & 200 & $100 \mathrm{~s}$ & 10 & FTP & 2 \\
\hline RTP & 200 & 100 & 20 & $\begin{array}{l}\text { Video } \\
\text { Conf. }\end{array}$ & 2 \\
\hline RTP & 200 & 100 & 10 & $\begin{array}{l}\text { Video } \\
\text { Conf. }\end{array}$ & 2 \\
\hline RTP & 60 & 40 & 20 & VOIP & 3 \\
\hline BE & 384 & N/A & N/A & HTTP & 2 \\
\hline
\end{tabular}

\section{RESULTS}

In this section, the output of simulation is shown and analyzed, the results shown in the section is measured in high congested environment were system load exceeds $140 \%$ of regular capacity

The first analyzed parameter, is the effect of Maximum latency on the throughput, Fig. 4, 5, 6, compare the performance of two session have the same MST, and mRR,but the they have difference maximum latency. The fist SS has 500ms maximum latency, while the second one is only limited for $100 \mathrm{~ms}$. Fig. 4 compares the performance if the two stations have high priority, it can be shown the two SS will have similar performance with a slight smoother output for the $100 \mathrm{~ms} \mathrm{SS}$. The main reason for this that the system is aiming to maximize the throughput, of this high priority traffic so normally it sent packets at intervals much smaller that maximum latency.

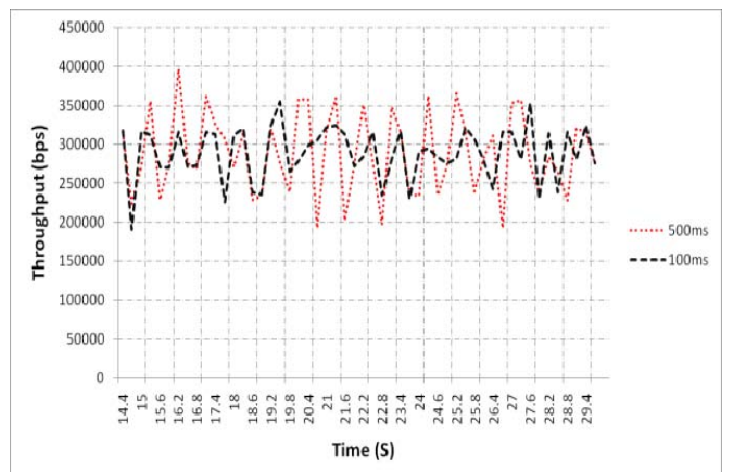

Fig. 4. Throughput of high priority traffic according to maximum delay 


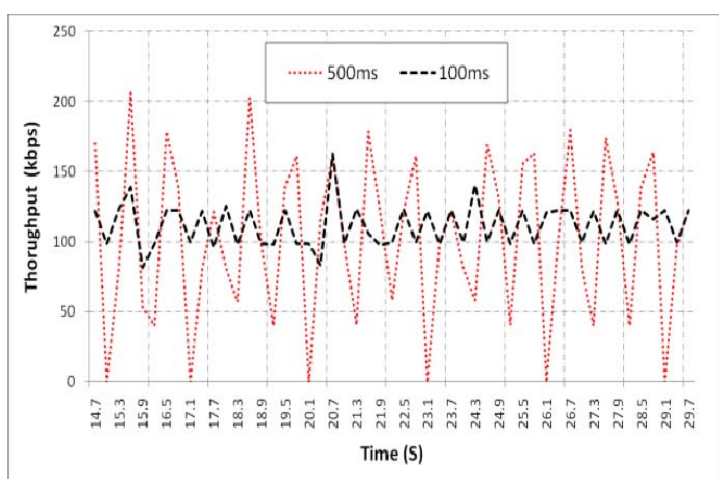

Fig. 5. Throughput of low priority traffic according to maximum delay

On the other hand, Fig. 5 shows the performance if the two SSs have lower priority. It can be shown that $100 \mathrm{~ms}$, SS has a very smooth output at very close intervals. However for the $500 \mathrm{~ms}$, it can shown that there is a big variance on system, throughput, mainly due the fact that the maximum latency is very high, $500 \mathrm{~ms}$, which allow longer periods without transmission as shown in the figure. Anyhow, it shows in Figure (6), that in both cases both stations were able to provide and average throughput of $100 \mathrm{Kbps}$, which equals to their $\mathrm{mRR}$ in this case.

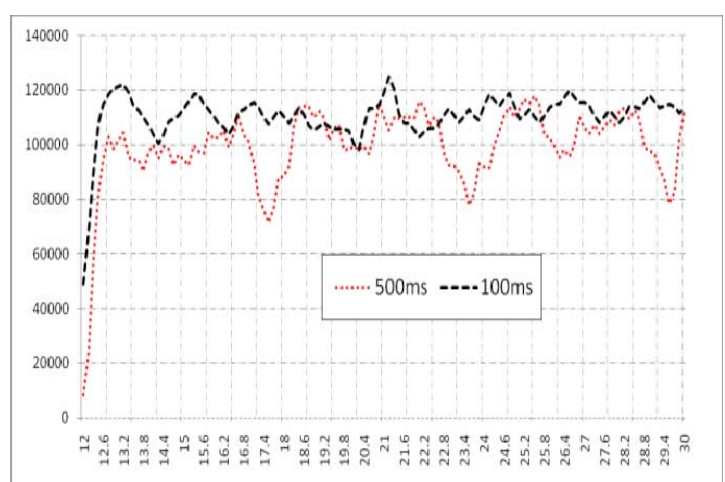

Fig. 6. Avg. Throughput of low priority traffic according to maximum delay

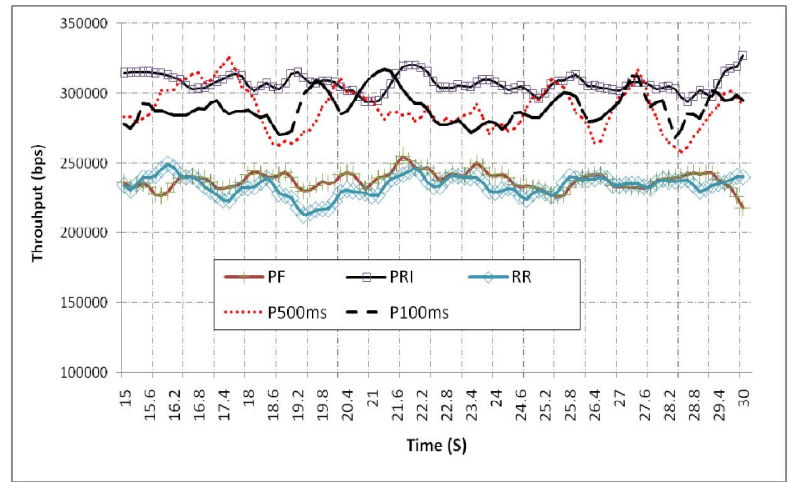

Fig. 7. AVG throughput of high priority traffic based on different algorithms

Fig. 7 compares the throughput of high priority traffic under the proposed algorithm, against other protocol, PF, $\mathrm{RR}$ and strict priority. It is shown from the figure that the proposed algorithm is capable to boost the performance of high priority traffic compared to PF and RR. The enhanced performance is achieved for both $100 \mathrm{~ms}$ delay and $400 \mathrm{~ms}$. Strict priority has a slightly higher performance since it dedicates all bandwidth for high-priority SS, while leaving less-priority SSs to starve as shown in Figure (8).

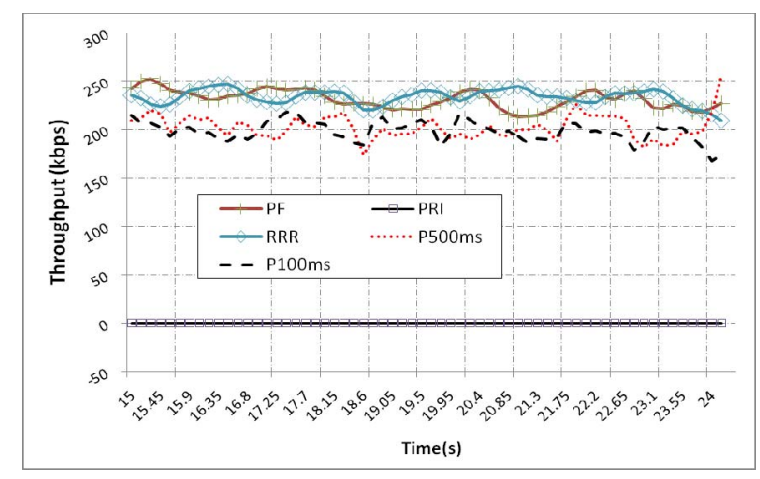

Fig. 8. AVG Throughput of low priority traffic based on different algorithms.

In Fig. 8, it can be shown that under low-priority traffic totally starve under strict priority algorithm. It also shown, the proposed algorithm is capable to meet the lower session constrains, which is $200 \mathrm{kbps}$ in this case. RR, PF have higher performance but this is on account of the throughput of high-priority traffic as shown in figure (7).

Finally, Fig. 9 shows packet delay variation of highpriority Voice over IP (VOIP)traffic under the proposed algorithm and is shown that it keeps within an acceptable range where a maximum variation is around $25 \mathrm{MS}$ at start of session

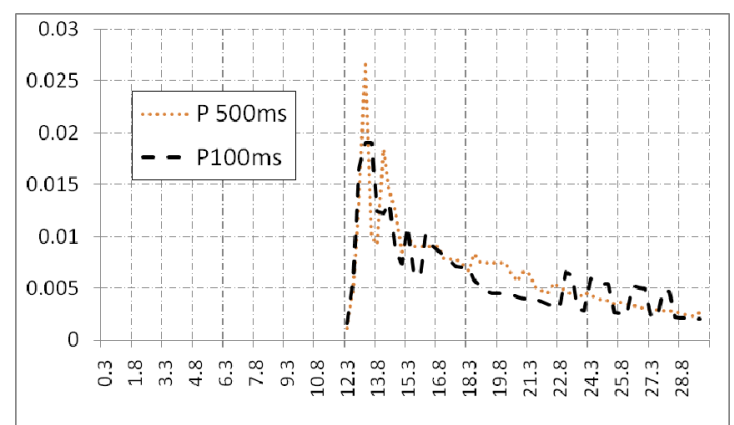

Fig. 9. Packet delay variation of VOIP session.

\section{CONCLUSIONS}

In this paper, a new scheduling algorithm for IEEE 802.16 Wireless MAN in PMP mode is proposed. The algorithm proposed a methodology to consider all the Qos constrains simultaneously. The algorithm is capable to differentiate throughput of various traffics based on their needs, including reserving the minimum throughput rate for low-priority traffic as well as boosting the traffic and performance of high priority traffic.

\section{REFERENCES}

[1] IEEE P802.16Rev2/D2, "DRAFT Standard for Local and metropolitan area networks," Part 16: Air Interface for Broadband Wireless Access Systems, 2007

[2] IEEE 802.16 Working Group on Broadband Wireless Access, http://wirelessman.org

[3] C. Eklude, R. B. Marks, K. L. Stanwood, and S. Wang, "IEEE Standard 802.16: A Technical Overview of the wireless MANTM Air Interface for Broadband Wireless Access," IEEE Communication Magazine June, 2002

[4] C. So-In, R. Jain, and A. K. Tamimi, "Scheduling in IEEE 802.16e Mobile WiMAX Networks: Key Issues and a Survey," IEEE Journal on Special Areas in Communications (JSAC), vol. 27, no. 2, 2009.

[5] L. Nuaymi, WiMAX, "Technology For Broadband Wireless Access," John Wiley and Sons, 2007. 
[6] L. Lin, W. Jia, and W. Lu, "Performance Analysis of IEEE 802.16 Multicast and Broadcast Polling based Bandwidth Request," in Proc. IEEE Wireless Communication and Networking Conf, Hong Kong, pp. 1854-1859. 2007.

[7] B. Chang and C. Chou, "Analytical Modeling of Contention-Based Bandwidth Request Mechanism in IEEE 802.16 Wireless Network," IEEE Trans. Veh. Technol, vol. 57, pp. 3094-3107, 2008.

[8] V. Alexey, Z. Ying, N. Qiang, and L. Andrey, "Efficient Request Mechanism Usage in IEEE 802.16," in Proc. IEEE Global Telecommunications Conf. San Francisco, pp. 1-5, 2006.

[9] V. Singh and V. Sharma, "Efficient and fair scheduling of plink and downlink in IEEE 802.16 OFDMA networks," Wireless Communications and Networking Conference, IEEE, 2006.
[10] A. H. Rashwan, H. M. El-Badawy, and H. H. Ali, "Comparative Assessments for Different WiMAX Scheduling Algorithms," pp. 20$22,2009$.

[11] C. Cicconetti, L. Lenzini, E. Mingozzi, and C. Eklund, "Quality of service support in IEEE 802.16 networks," IEEE Network, vol. 20, pp. $50-55,2006$.

[12] X. L. Huang and B. Bensaou, "On Max-min Fairness and Scheduling in Wireless Ad-Hoc Networks: Analytical Framework and Implementation," ACM MobiHoc'01, pp. 221-231, 2001.

[13] R. Chaijatupon and Y. Ji, "Simple Proportional Fairness Scheduling for OFDMA Frame-Based Wireless Systems," in Proc. IEEE Wireless Communication and Networking Conf, Las Vegas, pp. 1593120, 2008.

[14] www.Opnet.com 